\title{
F-7 - Proposal of the Polygonal Model for Representing Biomolecules as a Tool for Teaching Metabolism
}

\author{
Carlos Francisco Sampaio Bonafe ${ }^{1}$, José Ailton Conceição Bispo ${ }^{2}$, Marcelo Bispo de Jesus ${ }^{1}$ \\ ${ }^{1}$ Universidade Estadual de Campinas, Depto. de Bioquímica e Biologia Tecidual (Sao Paulo, Brasil); ${ }^{2}$ Universidade Estadual \\ de Feira de Santana, Tecnologia (Bahia, Brasil).
}

Support: FAPESP

\begin{abstract}
INTRODUCTION: Fischer projections are widely used for representing organic molecules, including the intermediates of metabolism. With the high number of molecules involving in metabolic processes and sometimes the relatively short time of learning of metabolism available for the students, it would be very useful an easier way to learn the structure of biomolecules and the involved reactions in metabolic pathways. OBJECTIVES: In this work it is presented the polygonal model, which consists in a new form of notation of biomolecules using geometric forms for representing the chemical groups in a biomolecule. MATERIALS AND METHODS: In this model, the carbonic chain is presented vertically using points of intersection or small black circles for carbon atoms, triangles, squares and circles represent, respectively, the hydroxyl, carbonyl and carboxyl groups, and the phosphate and amine groups are showed as, respectively, as yellow and blue circles. The stereoisomers and cis-trans isomers are clearly distinguishing as well. A form of familiarizing the student with the proposed notation can be to make an activity in class, furnishing a material which includes a short explanation of the model using examples, and some proposed activities of "transforming" molecules to polygonal model form from Fischer projections, and vice and versa. DISCUSSION AND RESULTS: It was observed that the average time necessary to complete this activity was less than $40 \mathrm{~min}$. Polygonal model found to have several advantages, as no loss of information of the molecule, the simplicity to show the reactions in a metabolic pathway, good receptivity by the students, capacity to diagnostic misconceptions of the students in organic chemistry. CONCLUSION: This method is much faster to represent the molecules. This is important in the dynamics of the class.
\end{abstract}

Keywords: Biomolecule representation; Polygonal model; metabolism teaching. 